\title{
MICB0106 gene polymorphism is associated with ulcerative colitis in central China
}

\author{
Yi Li $\cdot$ Bing Xia $\cdot$ Min Lü $\cdot$ Liuqing Ge $\cdot$ Xiaolian Zhang
}

Accepted: 22 July 2009 / Published online: 7 August 2009

(C) The Author(s) 2009. This article is published with open access at Springerlink.com

\begin{abstract}
Background The highly polymorphic nonclassical MHC class I chain-related genes $\mathrm{A}$ and $\mathrm{B}$ ( $M I C A$ and $M I C B)$ encode stress-inducible glycoproteins expressed on various epithelial cells including intestinal epithelial cells. MICA and MICB gene polymorphisms and expressions are associated with autoimmune diseases but not known in ulcerative colitis (UC).

Aims To investigate the association of MICB exon 2-4 polymorphisms and soluble MICA (sMICA) expression with the susceptibility of UC in central China.

Materials and methods Genomic DNA was isolated from peripheral blood. The allele frequencies of MICB exon 2-4 were genotyped in $105 \mathrm{UC}$ patients and 213 healthy controls by PCR single-stranded conformation polymorphism method.
\end{abstract}

Y. Li $\cdot$ B. Xia $\cdot$ M. Lü $\cdot$ L. Ge

Department of Gastroenterology and Research Center of Digestive

Diseases, Zhongnan Hospital,

Wuhan University School of Medicine,

Wuhan, People's Republic of China

Y. Li $\cdot$ B. Xia $\cdot$ M. Lü $\cdot$ L. Ge $\cdot$ X. Zhang

Clinical Research Center for Intestinal and Colorectal

Diseases and Key Laboratory of Allergy and Immune-related

Diseases of Hubei Province,

Wuhan, People's Republic of China

Y. Li

Department of Gastroenterology and Hepatology,

Erasmus Medical Center,

Rotterdam, The Netherlands

B. Xia $(\bowtie)$

Department of Gastroenterology,

Wuhan University Zhongnan Hospital,

Donghu Road 169,

Wuhan 430071, Hubei Province, People's Republic of China

e-mail: bingxia2004@yahoo.com.cn
Thirty-two patients and 32 controls were selected for determining serum sMICA expression by ELISA.

Results Allele frequency of MICB0106 was significantly higher in UC patients than in healthy controls $(19.0 \%$ vs. $8.9 \%$, corrected $P(P c)=0.0006)$, especially in patients with extensive colitis $(24.4 \%$ vs. $8.9 \%, P c=0.0006)$, moderate and severe disease $\left(24.1 \%\right.$ vs. $\left.8.9 \%, P_{c}=0.0006\right)$, extraintestinal manifestations $\left(20.5 \%\right.$ vs. $\left.8.9 \%, P_{c}=0.012\right)$, male patients $\left(22.1 \%\right.$ vs. $\left.8.0 \%, P_{c}=0.006\right)$, and patients over the age of 40 years $(28.8 \%$ vs. $8.3 \%, P c=0.0006)$. The sMICA level was significantly higher in UC than in healthy controls $(604.41 \pm 480.43 \mathrm{pg} / \mathrm{ml}$ vs. $175.37 \pm 28.31 \mathrm{pg} / \mathrm{ml}, P=0.0001)$ but not associated with the MICB0106 genotypes.

Conclusions Overall, MICB0106 allele was positively associated with UC in the Han Chinese in central China. sMICA was highly expressed in UC but not associated with the MICB0106 genotype.

Keywords MHC class I chain-related gene B .

Soluble MICA · Ulcerative colitis · Gene polymorphism

\section{Introduction}

Ulcerative colitis (UC) is a subtype of chronic nonspecific inflammatory bowel disease (IBD) with unknown etiology. Epidemiological studies in the European and American countries have demonstrated strong evidences of genetic susceptibility to IBD, such as concordance rates in twins and multiply-affected families $[1,2]$. Moreover, several genetic studies have found that the HLA genes, which are located on chromosome $6 p$ (IBD3), is one group of the susceptible loci of UC [3, 4]. Therefore, major histocompatibility complex (MHC) genes have been studied as candidate genes for the susceptibility of UC. 
The MHC class I chain-related (MIC) gene is located 47kilo base pairs $(\mathrm{kb})$ centromeric to HLA-B [5]. Mapping studies identified seven MIC loci (MICA-MICG), of which only MICA and MICB encode transcripts, while MICC, MICD, MICE, MICF, and MICG are pseudogenes. MIC molecules show homology with classical HLA molecules but do not combine with $\beta_{2}$ microglobulin or recognize antigen. The MIC molecules have three external domains $(\alpha 1-3)$, one transmembrane, and one cytoplasmic domain. The highly polymorphic MICA and MICB encode stress-inducible glycoproteins expressed on a variety of epithelial cells including intestinal epithelial cells. Interaction with the receptor NKG2D is likely to provide an important costimulatory signal for the activation of natural killer (NK) cells, macrophages, $\mathrm{CD} 8+\alpha \beta$, and $\gamma \delta$ T cells [6].

There are 65 recognized human MICA alleles and 30 different MICB alleles which have been identified so far (http://www.ebi.ac.uk/imgt/hla) [7]. The microsatellite polymorphism in exon 5 of MICA gene consists of five alleles based on the number of GCT/AGC triplet repeat units (alleles A4, A5, A6, and A9) and the presence of an additional nucleotide $\mathrm{G} / \mathrm{C}$ insertion (allele A5.1) [8]. MICB is the closest neighbor related to MICA, which has strict polymorphism. The $(\mathrm{CA} / \mathrm{TG}) \mathrm{n}$ microsatellite, consisting of 15 alleles, is present in intron 1 of the MICB gene (CA14-28) [9]. However, numerous high polymorphisms are more likely within the extracellular domain encoding exons 2, 3, and 4 of MICB. Recently, MICA and MICB gene polymorphisms have been found associated with autoimmune diseases [10] including insulin-dependent diabetes mellitus [11], Addison's disease [12, 13], celiac disease [14, 15], rheumatoid arthritis [16, 17], Behcet's disease [18, 19], and IBD [20-23], although little is known about the association between exons 2,3 , and 4 of MICB gene polymorphisms and UC.

In our previous studies, we found ethnic differences in the distribution of MICA (exon 5) and MICB (intron 1) gene polymorphisms [24-28] in central China. MICAA5.1/A5.1 homozygous genotype, MICA-A5.1 [27], and MICB-CA18 [28] allele are associated with UC in Chinese patients. Since MICA and MICB play important roles in immune regulation in autoimmune diseases and very few studies have observed an association of MICB polymorphism with the hereditary susceptibility to UC, therefore, we provided the exon 2-4 of MICB gene polymorphism and the soluble MICA (sMICA) expression for the susceptibility to UC in the Chinese Han population.

\section{Materials and methods}

Patients and healthy controls

A total of 105 UC patients with no family history who registered at our hospital from 2002 to 2006, were randomly collected and investigated. The diagnosis of UC was based on conventional clinical, endoscopic, and pathohistological criteria as described by Lennard-Jones [29]. Using the Montreal classification [30], the extent of the disease was classified as extensive colitis (involvement extends proximal to the splenic flexure) and left side colitis (involvement limited to a proportion of the colorectum distal to the splenic flexure) on endoscopy examination. The severity of UC was defined as mild, moderate, and severe colitis. We combined moderate and severe colitis since there were only five patients in the severe group. Extraintestinal manifestations (EIMs) of patients, including abnormal manifestations on joint, skin, eyes, and primary sclerosing cholangitis, were reviewed by records of the case history and interviews with the patients. A group of 213 unrelated ethnically matched blood donors who matched with gender and age were selected as healthy controls (HC). All the patients and controls were unrelated Chinese with Han nationality. The study was approved by the ethics committee of Wuhan University Zhongnan Hospital, and all the subjects gave informed consent.

\section{DNA isolation}

Genomic DNA was isolated from EDTA anti-coagulated peripheral venous blood by a conventional proteinase $\mathrm{K}$ digestion/phenol-chloroform extraction method.

\section{Allele genotyping for exon 2, 3, and 4 of MICB}

MICB alleles were determined by polymerase chain reaction (PCR) amplification on exons 2, 3, and 4 and followed by single-stranded conformation polymorphism (SSCP) analysis. The primers and annealing temperatures were forward 5'-CCAGGAAGAAGTTTCACCTG-3', reward 5'-CTGCTCTCTGTCTCCAACTT- $3^{\prime}, 54^{\circ} \mathrm{C}$; forward 5'-AAGGTGATGGGTTCGGGAAT-3', reward 5' - A GCAGAAT TGCGGGAACAGT- $3^{\prime}, \quad 56^{\circ} \mathrm{C}$; forward 5'-GTGAGAACAGTGGAGAGGAG-3', reward 5'-CACAGCCGTCCCTGCTGTT-3', 58 ${ }^{\circ} \mathrm{C}$, respectively, for amplifying exon 2, 3, and 4, which were designed using the genomic DNA sequence of MICB [31]. The concentration of primers used was $0.4 \mathrm{mM}$. PCR amplification was carried out in a $30-\mu \mathrm{L}$ volume containing $100 \mathrm{ng}$ of genomic DNA, $2.5 \mathrm{mM}$ of $\mathrm{MgCl}_{2}, 3 \mu \mathrm{L}$ $10 \times$ GeneAmp PCR Buffer II, $200 \mu \mathrm{M}$ of each dNTP, and 0.5 units of AmpliTaq Gold DNA Polymerase (PE Biosystems). The PCR conditions (in BIO-RAD PCR apparatus) were as follows: heating at $95^{\circ} \mathrm{C}$ for $5 \mathrm{~min}$, followed by 30 cycles of denaturation at $95^{\circ} \mathrm{C}$ for $30 \mathrm{~s}$, annealing temperature for $45 \mathrm{~s}$, extension at $72^{\circ} \mathrm{C}$ for $45 \mathrm{~s}$, and a final extension at $72^{\circ} \mathrm{C}$ for $10 \mathrm{~min}$. The amplified DNA fragments were analyzed by a previously described 
modified PCR-SSCP method [32]. Exons 2-4 were applied to an $8 \%$ polyacrylamide gel (without glycerol) and carried on electrophoresis at $200 \mathrm{~V}$ for $2 \mathrm{~h}$. Denatured singlestranded DNA fragments separated in the gel were visualized by silver staining. Genomic DNAs from HLA homozygous typing cell lines with MICB alleles were used as standards [33].

MICB alleles were assigned based on the distinct and combined SSCP patterns of exons 2, 3, 4, respectively (Fig. 1a-c). PCR products were directly sequenced as proved by an automated DNA sequencer (ABI PRISM 3730, BigDye terminator v3.1 reagent) using the same primers.

\section{sMICA analysis}

cMICA in serum was tested by sandwich enzymelinked immunosorbent assay (ELISA) [34]. The ELISA development kit contains the basic components required, including capture antibody, detection antibody, standard, and Streptavidin-HRP (R\&D Systems, USA). After being tested in microplate reader (Tecan, Australia) set at $450 \mathrm{~nm}$, we constructed a standard curve by plotting the mean absorbance for each standard on the $Y$ axis against the concentration on the $X$ axis, calculated as $\lg Y=-2.24555+0.66933 \times \lg X$. The data were fitted by plotting the log of the MICA levels (picograms per milliliter) versus the $\log$ of the O.D based on linear regression analysis.

\section{Statistical analysis}

SPSS 11.5 was used for statistical calculations. The allelic frequencies were calculated by direct counting, and statistical comparisons were performed by the $\chi^{2}$ test with Fisher's exact test. sMICA levels were analyzed by independent samples nonparametric test. Odds ratios (OR)

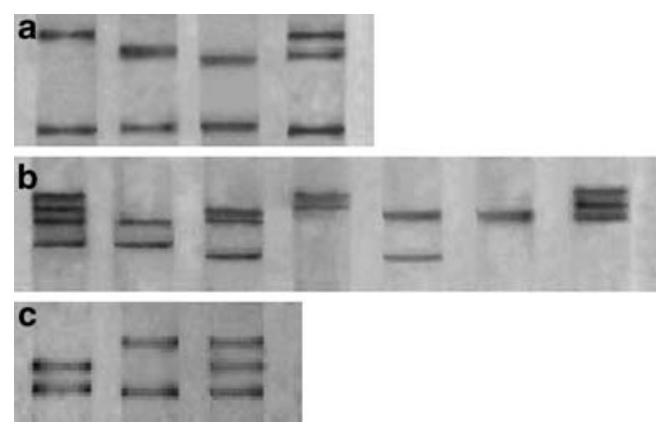

Fig. 1 PCR-SSCP analysis of MICB allele. a Three distinct banding patterns were observed in exon 2. b Four distinct banding patterns were observed in exon 3. c Only two distinct banding patterns were observed in exon 4. Banding patterns with one or two lanes showed homozygosis, while three or four lanes showed heterozygosis and $95 \%$ confidence intervals $(\mathrm{CI})$ were calculated for the carriers of the specific genotype. Bonferroni multiple corrections were used for the corrected $P(P c)$ by multiplying the $P$ value by the number of the statistical tests in order to avoid the $\alpha 1$ error. The $P$ and $P c$ below 0.05 were considered to be statistically significant.

\section{Results}

Baseline characteristics of UC patients

The demographic characteristics and clinical features of the patients are shown in Table 1.

No significant differences were found in gender and mean age of UC patients and controls (all $P>0.05$ ). In these 105 patients, the mean duration of UC was $(3.5 \pm 1.4)$ years. There were $41(39.0 \%)$ patients with extensive colitis and $64(61.0 \%)$ patients with left side colitis; $44(41.9 \%)$ patients had extraintestinal manifestation; 58 (55.2\%) patients suffered from with moderate or severe disease. Only one $(1.0 \%)$ patient had colostomy.

The distribution of MICB alleles between UC patients and healthy controls

The distribution of MICB alleles in 105 UC patients and 213 HC is shown in Table 2.

The frequency of MICB0106 was significantly higher in UC patients $(40 / 210)$ than in the healthy controls $(38 / 426$; $19.0 \%$ vs. $8.9 \% ; P=0.0001 ; P c=0.0006 ; O R=2.402 ; 95 \%$ CI, 1.488-3.879). Other differences between UC patients and healthy controls did not reach statistical significance.

The associations between the distribution of MICB alleles and clinical phenomenon of UC patients

When analyzing the distribution of MICB alleles in the subgroup of UC according to disease extent, severity, EIMs, and patients' gender and age, our study only showed that the frequency of MICB0106 was significantly higher in patients with extensive colitis $(20 / 82)$ than in those with left side colitis $(20 / 128 ; 24.4 \%$ vs. $8.9 \% ; P=0.0001 ; P c=$ 0.0006 ; $O R=3.294 ;$ 95\%CI, 1.800-6.027); higher in moderate and severe disease $(28 / 116)$ than in mild disease (12/ 94; $24.1 \%$ vs. $8.9 \% ; P=0.0001 ; P c=0.0006 ; O R=3.249$; 95\%CI, 1.893-5.576); and higher in those with EIMs (18/ $88)$ than in non-EIMs $(22 / 122 ; 20.5 \%$ vs. $8.9 \% ; P=0.002$; $P c=0.012 ; O R=2.626 ; 95 \% \mathrm{CI}, 1.418-4.861)$. Furthermore, the frequency of MICB0106 allele was significantly higher in the male patients $(27 / 122 ; 22.1 \%$ vs. $8.0 \% ; P=0.001$; $\left.P_{c}=0.006 ; O R=3.276 ; 95 \% \mathrm{CI}, 1.737-6.178\right)$ and in those patients over the age of 40 years $(34 / 118 ; 28.8 \%$ vs. $8.3 \%$; 
Table 1 Demographic characteristics and clinical features of the patients with UC and healthy controls

\begin{tabular}{lrr}
\hline & UC $(n=105)$ & HC $(n=213)$ \\
\hline Gender (\%) & $61(58.1 \%)$ & $119(55.9 \%)$ \\
Male & $44(41.9 \%)$ & $44(44.1 \%)$ \\
Female & $3.5 \pm 1.4(1-18)$ & \\
Mean age of diagnosis (range, in years) & $46.95 \pm 14.01(16-79)$ & \\
Mean course of disease (years) & $41(39.0 \%)$ & \\
Extent of disease (\%) & $64(61.0 \%)$ & \\
Extensive colitis & & \\
Left side colitis & $44(41.9 \%)$ & \\
Extraintestinal manifestations $(\%)$ & $61(58.1 \%)$ & \\
Yes & & \\
No & & \\
Severity of disease $(\%)$ & $58(55.2 \%)$ & \\
Mild & $1(1.0 \%)$ & \\
Moderate and severe &
\end{tabular}

$P=0.0001 ; P c=0.0006 ; O R=4.500 ; 95 \%$ CI, 2.381-8.504) compared with healthy controls (13/88 and $17 / 206$, respectively). Therefore, MICB0106 allele was associated with extensive colitis, moderate and severe disease, EIMs, male UC patients, and the patients over the age of 40 years.

The difference in MICB distributions between China and western countries

To determine the effect of ethnicity on MICB gene polymorphism and even its susceptibility to UC, we also investigated the differences in MICB distributions between China (our study) and western countries [22, 35] (Table 3). There were significant differences in MICB distribution between the Chinese and English $(P=0.0001)$ and between the Chinese and the Spanish $(P=0.018)$.

sMICA expression in UC patients and healthy controls

In order to investigate sMICA, we selected $33 \mathrm{UC}$ patients and 32 healthy controls randomly from the subjects we had previously. The patients were divided into three subgroups: (1) $12 \mathrm{UC}$ patients carried
MICB0106; (2) Eight UC patients carried MICA-A5.1; (3) 13 UC patients carried neither MICB0106 nor MICA-A5.1. Those who carried both MICB0106 and MICA-A5.1 were not included. Tested by a sandwich ELISA, the mean sMICA concentration in the serum of 33 UC patients $(604.41 \pm 480.43 \mathrm{pg} / \mathrm{ml})$ was 3 6-fold higher than that in 32 healthy control $(175.37 \pm$ $28.31 \mathrm{pg} / \mathrm{ml}$; Fig. 2a). The significant difference reached $Z=-6.967, P=0.0001$, which indicates that sMICA was strongly associated with UC.

The correlation between sMICA expression and MICB gene polymorphism

To correlate sMICA expression to MICB gene polymorphism, we compared the three intersubgroups of UC. The sMICA concentration in both subgroups of MICB0106 carriers and MICA-A5.1 carriers were higher than those in non-MICB0106/MICA-A5.1 carriers $(647.64 \pm 518.07 \mathrm{pg} /$ $\mathrm{ml}$ vs. $543.32 \pm 343.70 \mathrm{pg} / \mathrm{ml} ; 641.76 \pm 603.73 \mathrm{pg} / \mathrm{ml}$ vs. $543.32 \pm 343.70 \mathrm{pg} / \mathrm{ml}$ ), but the differences between sMICA levels among them did not reach statistical significance $(P=0.487>0.05)($ Fig. 2b)
Table 2 MICB allele frequencies in patients with ulcerative colitis (UC) and healthy controls (HC) $[n(\%)]$

\begin{tabular}{lccccr}
\hline MICB allele & UC $(2 n=210 ; \%)$ & HC $(2 \mathrm{n}=426 ; \%)$ & $\chi^{2}$ & $P$ & $P c$ \\
\hline 0102 & $86(41.0)$ & $189(44.4)$ & 0.668 & 0.414 & 2.484 \\
0103 & $28(13.3)$ & $53(12.4)$ & 0.101 & 0.751 & 4.506 \\
0104 & $32(15.2)$ & $72(16.9)$ & 0.285 & 0.594 & 3.564 \\
0105 & $12(5.7)$ & $43(10.1)$ & 3.415 & 0.065 & 0.390 \\
0106 & $40(19.0)^{\mathrm{a}}$ & $38(8.9)$ & 13.408 & 0.0001 & $<0.001$ \\
$0107 \mathrm{~N}$ & $12(5.7)$ & $31(7.3)$ & 0.545 & 0.460 & 2.760 \\
\hline
\end{tabular}

${ }^{\mathrm{a}} \mathrm{OR}=2.402 ; 95 \% \mathrm{CI}$, $1.488-3.879$ 
Table 3 The difference of MICB distributions in China and western countries

\begin{tabular}{lccc}
\hline MICB allele & Hubei Han Chinese & English & Spanish \\
\hline$\chi^{2}$ & - & 50.233 & 13.700 \\
Degrees of freedom & - & 5 & 5 \\
$P$ & - & 0.0001 & 0.018 \\
\hline
\end{tabular}

\section{Discussion}

Our study focused on the polymorphism of MIC gene which has strong linkage disequilibrium with MHC class I gene. MICA and MICB exclusively encode transcripts and respond to protein expression among all MIC members, while the association of MICB exon 2-4 polymorphisms and soluble MICA (sMICA) expression with the susceptibility of UC has still not been clarified.

In recent studies on MIC genes susceptible to UC, MICA*007 [23], MICA*00801, and MICA-A6 [36] were shown to be associated with UC onset in Japan. Previously, we found that MICA-A5.1/A5.1 homozygous genotype MICA-A5.1 [27] and MICB-CA18 [28] alleles were associated with UC in the Chinese Han population. In this study, we included a limited population in central China which may not be generalized; however, we found that the frequency of MICB0106 allele was significantly higher in the UC group, especially in patients with extensive colitis, moderate and severe disease, and in those with extraintestinal manifestations. In addition, the frequency of MICB0106 was significantly higher in the male UC patients and patients over the age of 40 years than in healthy controls. These positive associations suggest that the people carrying MICB0106 allele are at higher risk of developing UC and might be affected more severely and more extensively throughout the colon or develop more extraintestinal manifestations. In terms of UC susceptibility, males and older people are more affected. We did not analyze the association of UC with MICB genotype because of the small size of each subgroup.

So far, very few studies have observed a relationship between MICB gene polymorphism and UC. Based on genotyping, we selected several publications [22, 35] from a number of different ethnic populations. The comparison of MICB alleles in our study (Hubei Han population, China) with those in England and Spain showed significant differences in the distribution of the exon2-4 of MICB alleles (Chinese vs. English, $\chi^{2}=50.233, P=0.0001$; Chinese vs. Spanish, $\chi^{2}=13.700, P=0.018$ ). Furthermore, significant differences in the distribution of the intron 1 of MICB alleles were also observed between the Chinese and other populations [28]. These findings suggest that the differences in ethnic and regional living environment would influence on the $M I C B$ gene distribution. The association of MICB with UC might be specific to the Chinese population or even to the Han population in central China.

The neighbors MICA and MICB have big similarities in both molecular structure and encoding function. The analysis in available Spanish families indicated that MICB0106 was part of the B8-DR3-DQ2 haplotype (HLA-B*0802-MICA-A5.1-MICB0106-DRB1-*0301DQA1-*0501-DQB1-*0201). Accordingly, a strong linkage disequilibrium of MICB0106 with DQ2 $(\lambda s=1)$ and MICAA5.1 $(\lambda s=0.55)$ was observed [37]. In our previous study [27], we also observed that some MICA-MICB haplotypes occurred at frequencies of strong linkage disequilibrium $(P<0.01)$. Therefore, we hypothesize that sMICA expression in serum might also be associated with UC.

By ELISA analysis, we found sMICA are positively associated with UC compared with healthy controls but not associated with MICB gene polymorphism. These observations indicate that sMICA plays a very important role in UC patients, while it is still not clear whether sMICA protein

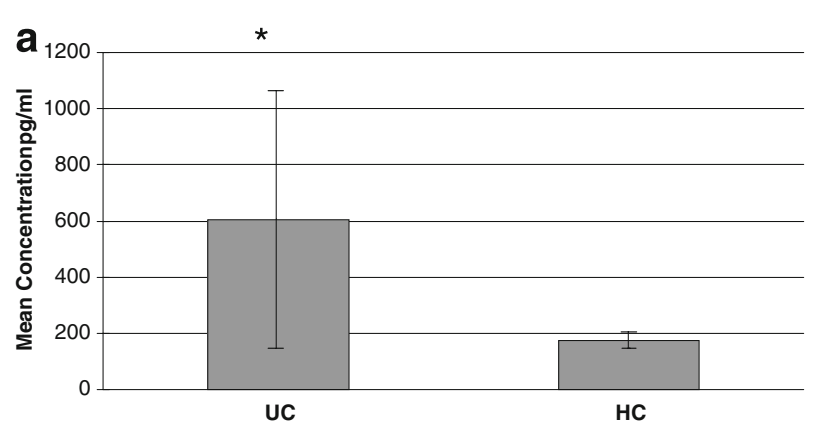

Fig. 2 a sMICA concentration in UC patients and HC.* The mean sMICA concentration in the serum of 33 UC patients $(604.41 \pm$ $480.43 \mathrm{pg} / \mathrm{ml}$ ) was significantly higher than that in 32 healthy controls $(175.37 \pm 28.31 \mathrm{pg} / \mathrm{ml}) . Z=-6.967, P=0.0001$. b sMICA concentration in three subgroups of UC patients. The sMICA concentration in the

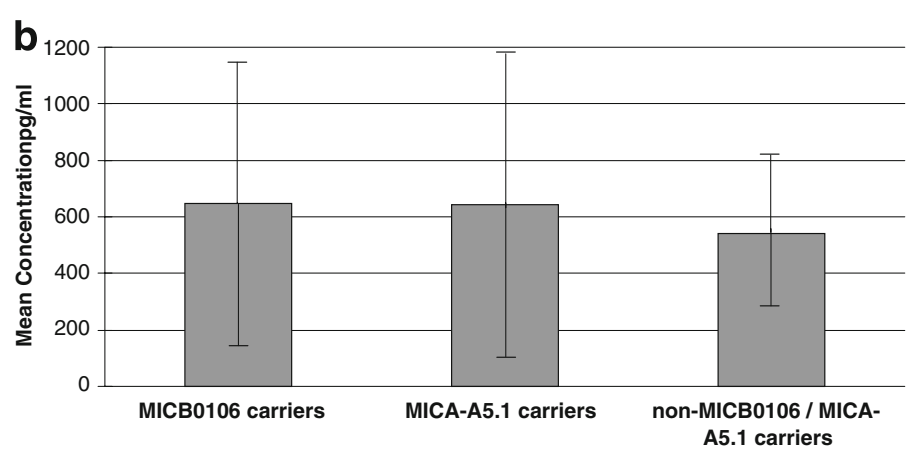

subgroups of MICB0106 carriers, MICA-A5.1 carriers, and nonMICB0106/MICA-A5.1 carriers was $647.64 \pm 518.07 \mathrm{pg} / \mathrm{ml}, 641.76 \pm$ $603.73 \mathrm{pg} / \mathrm{ml}$, and $543.32 \pm 343.70 \mathrm{pg} / \mathrm{ml}$, respectively. The differences between sMICA levels among them did not reach statistical significance $(P=0.487>0.05)$ 
expression is related to the MICB0106 and MICA-A5.1 genotype. The significantly higher MICB0106 gene expression may not be able to fully affect sMICA protein expression, which means that it might affect the function of MIC genes in ways other than genotype or the relatively small sample we studied. A bias of this study might also be that the serum samples were not taken at the time point of diagnosis, so that the rate of disease development may vary between patients. Therefore, a prospective study to analyze serum samples procured at the time of diagnosis will help to clarify whether sMICA can identify risk patients or optimize patient treatment.

As previously reported, MIC expression is under the control of heat shock elements and is increased in response to cellular stress [6] including viral [38] and bacterial infection [39]. In recent observations, MIC-NKG2D engagement augments both the cytotoxic response of CMVinfected $\mathrm{CD} 8+\alpha \beta$ T cells and TCR-dependent activation of $\mathrm{V} \gamma 2 \mathrm{~V} \delta 2 \mathrm{~T}$ cells by bacterial antigens, which suggest that this interaction might provide an important costimulatory signal for cytokine production and cellular proliferation [22]. sMICA molecules impair the functions of NKG2D+ T and NK cells, which may facilitate tumor cells to escape from immunosurveillance [40]. Serum levels of sMICA have been shown to be of diagnostic significance in malignant diseases of various origins, including multiple myeloma [41] and colon adenocarcinoma [34]. sMICA are elevated and responsible for down-modulation of NKG2D and other important activating chemokine receptors to deactivate NK immunity. These immune reactions may also present in UC disease which confirms our findings.

In conclusion, our study strongly suggests that MICB gene polymorphism is positively associated with UC in the Han Chinese in central China; sMICA was highly expressed in UC but not associated with the MICB0106 genotype. Further investigation on the function of MIC genes may be interesting in future.

Acknowledgment This project was supported financially by grants from the Natural Science Foundation of China (30570834) and Ministry of Public Health (200802156). The study was approved by the ethics committee of Wuhan University Zhongnan Hospital, and all the subjects gave informed consents.

Open Access This article is distributed under the terms of the Creative Commons Attribution Noncommercial License which permits any noncommercial use, distribution, and reproduction in any medium, provided the original author(s) and source are credited.

\section{References}

1. Karlinger K, Gyorke T, Mako E et al (2000) The epidemiology and the pathogenesis of inflammatory bowel disease. Eur J Radiol 35:154-167
2. Binder V, Orholm M (1996) Familial occurrence and inheritance studies in inflammatory bowel disease. Neth J Med 48:53-56

3. Dechairo B, Dimon C, van Heel D et al (2001) Replication and extension studies of inflammatory bowel disease susceptibility regions confirm linkage to chromosome 6p (IBD3). Eur J Hum Genet 9:627-633

4. Brant SR, Shugart YY (2004) Inflammatory bowel disease gene hunting by linkage analysis: rationale, methodology, and present status of the field. Inflamm Bowel Dis 10:300-311

5. Bahram S, Spies T (1996) The MIC gene family. Res Immunol 147:328-333

6. Groh V, Steinle A, Bauer S et al (1998) Recognition of stressinduced MHC molecules by intestinal epithelial gamma delta $\mathrm{T}$ cells. Science 279:17-40

7. Robinson J, Waller MJ, Parham P et al (2003) IMGT/HLA and IMGT/MHC: sequence databases for the study of the major histocompatibility complex. Nucleic Acids Res 31:311-314

8. Ota M, Katsuyama Y, Mizuki N et al (1997) Trinucleotide repeat polymorphism within exon 5 of the MICA gene (MHC class I chain-related gene A): allele frequency data in the nine population groups Japanese, Northern Han, Hui, Uygur, Kazakhstan, Iranian, Saudi Arabian, Greek and Italian. Tissue Antigens 49:448-454

9. Glas J, Martin K, Brunnler G et al (2001) MICA, MICB and C1_4_1 polymorphism in Crohn's disease and ulcerative colitis. Tissue Antigens 58:243-249

10. Bilbao JR, Martin-Pagola A, De Perez NG et al (2003) HLADRB1 and MICA in autoimmunity: common associated alleles in autoimmune disorders. Ann N Y Acad Sci 2003(1005):314-318

11. Gambelunghe G, Ghaderi CA et al (2000) Association of MHC Class I chain-related A (MIC-A) gene polymorphism with Type I diabetes. Diabetologia 43:507-514

12. Park YS, Sanjeevi CB, Robles D et al (2002) Additional association of intra-MHC genes, MICA and D6S273, with Addison's disease. Tissue Antigens 60:155-163

13. Gambelunghe G, Falorni A, Ghaderi M et al (1999) Microsatellite polymorphism of the MHC class I chain-related (MIC-A and MIC-B) genes marks the risk for autoimmune Addison's disease. J Clin Endocrinol Metab 84:3701-3707

14. Rueda B, Pascual M, Lopez-Nevot MA et al (2003) Association of MICA-A5. 1 allele with susceptibility to celiac disease in a family study. Am J Gastroenterol 98:359-362

15. Rodriguez-Rodero S, Rodrigo L, Fdez-Morera JL et al (2006) MHC class I chain-related gene B promoter polymorphisms and celiac disease. Hum Immunol 67:208-214

16. Mok JW, Lee YJ, Kim JY et al (2003) Association of MICA polymorphism with rheumatoid arthritis patients in Koreans. Hum Immunol 64:1190-1194

17. Lopez-Arbesu R, Ballina-Garcia FJ, Alperi-Lopez M et al (2007) MHC class I chain-related gene B (MICB) is associated with rheumatoid arthritis susceptibility. Rheumatology 46:426-430

18. Salvarani C, Boiardi L, Mantovani V et al (2001) Association of MICA alleles and HLA-B51 in Italian patients with Behcet's disease. J Rheumatol 28:1867-1870

19. Hughes EH, Collins RW, Kondeatis E et al (2005) Associations of major histocompatibility complex class I chain-related molecule polymorphisms with Behcet's disease in Caucasian patients. Tissue Antigens 66:195-199

20. Seki SS, Sugimura K, Ota M et al (2001) Stratification analysis of MICA triplet repeat polymorphisms and HLA antigens associated with ulcerative colitis in Japanese. Tissue Antigens 58:71-76

21. Fdez-Morera JL, Rodrigo L, Lopez-Vazquez A et al (2003) MHC class I chain-related gene A transmembrane polymorphism modulates the extension of ulcerative colitis. Hum Immunol 64:816-822

22. Ahmad T, Marshall SE, Mulcahy-Hawes K et al (2002) High resolution MIC genotyping: design and application to the 
investigation of inflammatory bowel disease susceptibility. Tissue Antigens 60:164-179

23. Sugimura K, Ota M, Matsuzawa J et al (2001) A close relationship of triplet repeat polymorphism in MHC class I chain-related gene A (MICA) to the disease susceptibility and behavior in ulcerative colitis. Tissue Antigens 57:9-14

24. Lü M, Xia B (2006) Polymorphism of HLA-DRB1 gene shows no strong association with ulcerative colitis in Chinese patients. Int $\mathbf{J}$ Immunogenet 33:37-40

25. Xia B, Crusius JB, Wu J et al (2002) CTLA4 gene polymorphisms in Dutch and Chinese patients with inflammatory bowel disease. Scand J Gastroenterol 37:1296-1300

26. Xia B, Crusius JB, Wu J et al (2003) Signal transducer and activator of transcription 6 gene G2964A polymorphism and inflammatory bowel disease. Clin Exp Immunol 131:446-450

27. Ding YJ, Xia B, Lü M et al (2005) MHC class I chain-related geneA-A5.1 allele is associated with ulcerative colitis in Chinese population. Clin Exp Immunol 142:193-198

28. Lü M, Xia B, Li J et al (2006) MICB microsatellite polymorphism is associated with ulcerative colitis in Chinese population. Clin Immunol 120:199-204

29. Lennard-Jones JE (2006) Classification of inflammatory bowel disease. Scand J Gastroenterol 24:2-6

30. Satsangi J, Silverberg MS, Vermeire S et al (2006) The Montreal classification of inflammatory bowel disease: controversies, consensus, and implications. Gut 55:749-753

31. Bahram S, Shiina T, Oka A et al (1996) Genomic structure of the human MHC class I MICB gene. Immunogenetics 45:161-162

32. Bannai M, Tokunaga K, Lin L et al (1994) Discrimination of human HLA-DRB1 alleles by PCR-SSCP (single-strand conformation polymorphism) method. Eur J Immunogenet 21:1-9
33. Ando H, Mizuki N, Ota M et al (1997) Allelic variants of the human MHC class I chain-related B gene $(M I C B)$. Immunogenetics 46:449-508

34. Ekaterina SD, Mikhail MD, Elena V et al (2003) Evasion from NK cell immunity by MHC class I chain-related molecules expressing colon adenocarcinoma. J Immunol 78: 6891-6899

35. González S, Rodrigo L, López-Vázquez A et al (2004) Association of MHC class I related gene B (MICB) to celiac disease. Am J Gastroenterol 99:676-680

36. Ahmad T, Armuzzi A, Neville M et al (2003) The contribution of human leucocyte antigen complex genes to disease phenotype in ulcerative colitis. Tissue Antigens 62:527-35

37. Gonzalez S, Rodriguez-Rodero S, Martinez-Borra J et al (2003) MICB typing by PCR amplification with sequence specific primers. Immunogenetics 54:850-855

38. Groh V, Rhinehart R, Randolph-Habecker J et al (2001) Costimulation of $\mathrm{CD} 8 \mathrm{aB}$ T cells by NKG2D via engagement by MIC induced on virus-infected cells. Nat Immunol 2:255260

39. Das H, Groh V, Kuijl C et al (2001) MICA engagement by human $\mathrm{V} 12 \mathrm{Vd} 2 \mathrm{~T}$ cells enhances their antigen-dependant effector function. Immunity 15:83-93

40. Holdenrieder S, Stieber P, Peterfi A et al (2006) Soluble MICB in malignant diseases: analysis of diagnostic significance and correlation with soluble MICA. Ca Immunol Immunother 55:1584-1589

41. Rebmann V, Schutt P, Brandhorst D et al (2007) Soluble MICA as an independent prognostic factor for the overall survival and progression-free survival of multiple myeloma patients. Clin Immunol 123:114-120 